\title{
Molecular Mechanisms Underlying Anti-Inflammatory Actions of 6-(Methylsulfinyl)hexyl Isothiocyanate Derived from Wasabi (Wasabia japonica)
}

\author{
Takuhiro Uto, ${ }^{1}$ De-Xing Hou, ${ }^{2}$ Osamu Morinaga, ${ }^{1}$ and Yukihiro Shoyama ${ }^{1}$ \\ ${ }^{1}$ Department of Pharmacognosy, Faculty of Pharmaceutical Sciences, Nagasaki International University, \\ 2825-7 Huis Ten Bosch, Sasebo, Nagasaki 859-3298, Japan \\ ${ }^{2}$ Course of Biological Science and Technology, United Graduate School of Agricultural Sciences, Kagoshima University, \\ Korimoto 1-21-24, Kagoshima 890-0065, Japan
}

Correspondence should be addressed to Takuhiro Uto, uto@niu.ac.jp

Received 25 May 2012; Accepted 15 July 2012

Academic Editor: Alessandra Bitto

Copyright (c) 2012 Takuhiro Uto et al. This is an open access article distributed under the Creative Commons Attribution License, which permits unrestricted use, distribution, and reproduction in any medium, provided the original work is properly cited.

\begin{abstract}
6-(Methylsulfinyl)hexyl isothiocyanate (6-MSITC) is a major bioactive compound in wasabi (Wasabia japonica), which is a typical Japanese pungent spice. Recently, in vivo and in vitro studies demonstrated that 6-MSITC has several biological properties, including anti-inflammatory, antimicrobial, antiplatelet, and anticancer effects. We previously reported that 6-MSITC strongly suppresses cyclooxygenase-2 (COX-2), inducible nitric oxide synthase (iNOS), and cytokines, which are important factors that mediate inflammatory processes. Moreover, molecular analysis demonstrated that 6-MSITC blocks the expressions of these factors by suppressing multiple signal transduction pathways to attenuate the activation of transcriptional factors. Structure-activity relationships of 6-MSITC and its analogues containing an isothiocyanate group revealed that methylsulfinyl group and the length of alkyl chain of 6-MSITC might be related to high inhibitory potency. In this paper, we review the anti-inflammatory properties of 6-MSITC and discuss potential molecular mechanisms focusing on inflammatory responses by macrophages.
\end{abstract}

\section{Introduction}

Isothiocyanates (ITCs) are a group of naturally occurring sulfur compounds containing $-\mathrm{N}=\mathrm{C}=\mathrm{S}$ functional group, available often abundantly from many cruciferous vegetables. ITCs are stored as glucosinolate precursors in the plants. The damage of plant tissue such as chopping and mastication activates myrosinase which hydrolyses the glucosinolate (myrosinase-glucosinolate system), and the resultant ITCs play a key role in the defense against herbivores and pathogens $[1,2]$. There are a significant number of naturally occurring and synthetic ITCs, and numerous studies have demonstrated the chemopreventive and anti-inflammatory properties of ITCs in vitro and in vivo [3-5]. Accumulating evidence suggests that ITCs exert their effects through a variety of signaling pathways involved in detoxification, inflammation, apoptosis, and cell cycle regulation, among others [4-6].
Wasabi (Wasabia japonica) is a member of the Brassicaceae family of vegetables, and its rhizome is a very popular pungent spice in Japan. Several studies have shown that wasabi has multiple physiological functions, such as appetite enhancement [7], antimicrobial activity [8], inhibition of platelet aggregation [9], and the suppression of $N$-methyl- $N^{\prime}$-nitro- $N$-nitrosoguanidine-induced rat gastric carcinogenesis [10]. Wasabi differs from other Brassicaceae species in that it contains higher concentration of ITCs, especially long-chain ITCs. The bioactive components of wasabi have been identified as a series of ITC analogues, of which 6-(methylsulfinyl)hexyl isothiocyanate (6-MSITC or 6-MITC) (Figure 1) is a major active compound in wasabi. Several lines of evidence demonstrated the pharmacological potencies of 6-MSITC, such as anti-inflammatory [1113], antimicrobial [14], antiplatelet [15], and anticancer [16-18] effects. We previously reported that 6-MSITC strongly suppresses inflammatory mediators by regulating 


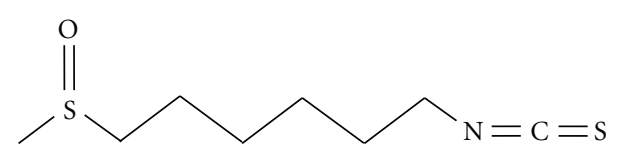

Figure 1: Chemical structure of 6-MSITC. 6-MSITC contains methyl sulfoxide group and ITC group linked by alkyl chain.

the signaling pathways [11-13]. In this paper, we describe the anti-inflammatory properties of 6-MSITC and discuss potential molecular mechanisms with special attention to several inflammatory factors in macrophages.

\section{Chemistry and Extraction of 6-MSITC}

A number of analogues of ITCs are isolated from wasabi, and the main pungent compound of wasabi is allyl ITC. Ina et al. [19] and Etoh et al. [20] reported that the characteristic flavor of wasabi depends on the methylthioalkyl ITCs and methylsulfinylalkyl ITCs. The methylthioalkyl ITCs are found in wasabi alone, whereas methylsulfinylalkyl ITCs are found in both wasabi and horseradish. Wasabi contains much higher levels of all methylsulfinylalkyl ITCs than horseradish [19]. One of the methylsulfinylalkyl ITCs is 6MSITC, and the chemical structure of 6-MSITC contains methyl sulfoxide group and ITC group linked by alkyl chain (Figure 1). Although the ITCs are generated throughout the entire plant of wasabi, the root is the predominant site of storage. Hara et al. [21] found the myrosinase-glucosinolate composition of wasabi is in the epidermis and vascular cambium of the root. Indeed, wasabi root is particularly rich in most ITCs [19].

Several groups isolated 6-MSITC from wasabi root by monitoring the cellular bioactivities. Ono et al. [22] purified 6-MSITC from the water-soluble fractions of wasabi by monitoring the growth inhibition of MKN-28 cells. The watersoluble fraction of wasabi roots was fractionated by Sephadex G-15 gel filtration and reverse-phase HPLC successfully, and active compound was finally collected by preparative HPLC. The spectroscopic data, including fast atom bombardment mass spectrometry (FAB-MS) and electron ionization mass spectrometry (EI-MS), determined a molecular ion peak at $[\mathrm{M}]^{+} \mathrm{m} / z 205$ and the molecular formula of $\mathrm{C}_{8} \mathrm{H}_{15} \mathrm{NOS}_{2}$. Furthermore, IR and NMR completely confirmed that an active compound is 6-MSITC. Morimitsu et al. [23] extracted the smashed wasabi root to give an active ethyl acetate fraction, resulting in 6-MSITC as the major active compound of glutathione $S$-transferase (GST) activity after purification by silica gel column chromatography and preparative HPLC. The content of 6 -MSITC in wasabi is $\sim 550-556 \mu \mathrm{g} / \mathrm{g}$ wet body weight of wasabi root.

\section{Effect of 6-MSITC on Inflammatory Factors}

Inflammation is one of the most important host defense systems against tissue injuries and pathogen invasion [24]. In the inflammatory process, macrophages play a central role in induction of inflammatory enzymes, cytokines, chemokines, and other inflammatory factors. Overexpression of these inflammatory factors by macrophages has been implicated in the pathophysiology of many inflammatory diseases, such as rheumatoid arthritis, atherosclerosis, chronic hepatitis, pulmonary fibrosis, and inflammatory brain diseases [25, 26]. Lipopolysaccharide (LPS), a component of Gramnegative bacterial cell wall, activates macrophages to produce prostaglandin $\mathrm{E}_{2}\left(\mathrm{PGE}_{2}\right)$ by cyclooxygenase-2 (COX-2), nitric oxide (NO) by inducible NO synthase (iNOS), and inflammatory cytokines through the activating multiple signaling pathways $[27,28]$. Thus, the biological reduction of LPS-inducible inflammatory factors is considered to be an effective strategy for inflammatory diseases.

3.1. COX-2. COXs catalyze the synthesis of prostaglandins from arachidonic acid. There are two isoforms of COX, designated COX-1 and COX-2, which are encoded by different genes. COX-1 is constitutively expressed in most tissues and believed to be responsible for normal physiological functions [29]. In contrast, COX-2 is not detectable in normal tissues or resting immune cells, but it could be induced by LPS, inflammatory cytokines, growth factors, and carcinogens $[30,31]$.

6-MSITC suppressed LPS-induced COX-2 expression and $\mathrm{PGE}_{2}$ release in murine macrophage cell lines RAW264 and human U937 monocytic cells without affecting the constitutive COX-1 expression [11]. Molecular analysis demonstrated that 6-MSITC blocked LPS-induced COX2 expression in transcriptional level. In the COX-2 gene, cis-acting elements including nuclear factor $\kappa \mathrm{B}$ (NF$\kappa \mathrm{B})$, CCAAT/enhancer-binding protein $(\mathrm{C} / \mathrm{EBP})$, and cyclic AMP-response element (CRE) have been identified to play a critical role in regulating transcription [32-36]. Moreover, single site of NF- $\kappa \mathrm{B}, \mathrm{C} / \mathrm{EBP}$, or CRE cannot sufficiently respond to induce COX-2 transcription activity, and two of these cis-acting elements are at least recruited to achieve maximal induction of transcription [32]. 6-MSITC inhibited LPS-induced COX-2 expression by suppressing transcriptional factors binding to the first 327 base pairs in the $5^{\prime}$ flanking regions of COX-2 gene [11]. Moreover, mutation of a single NF- $\kappa \mathrm{B}, \mathrm{C} / \mathrm{EBP}$, or CRE promoter element did not abrogate the effect of 6-MSITC. Thus, the inhibition of at least two of these cis-elements is required to achieve the maximal inhibitory action of 6-MSITC on COX-2 gene expression, suggesting that the inhibitory effect of 6-MSITC on COX-2 expression could be obtained by targeting the signaling pathways leading to at least two promoter elements including NF- $\kappa \mathrm{B}, \mathrm{C} / \mathrm{EBP}$, and CRE sites.

In mouse RAW264 macrophages, COX-2 expression was activated by interferon (IFN)- $\gamma$ and 12-Otetradecanonoylphorbol-13-acetate (TPA) in the same manner as LPS [13]. Interestingly, 6-MSITC downregulated COX-2 expression induced by LPS and IFN $-\gamma$ but did not suppress that induced by TPA. These data indicated that LPS, IFN- $\gamma$, and TPA regulate COX-2 expression through different pathways, and 6-MSITC acts as a potent inhibitor against LPS- or IFN- $\gamma$-induced COX-2 expression. 
3.2. iNOS. $\mathrm{NO}$ is produced endogenously during arginine metabolism by isoforms of NOS $[37,38]$. NO has a number of important biological functions, including tumor cell killing, host defense against intracellular pathogens, neurotransmission, and inhibition of platelet aggregation [39]. However, excess $\mathrm{NO}$ is a potent mediator and regulator of inflammatory responses $[40,41]$ and also has a multifaceted role in process of cancer [42]. NO is synthesized from Larginine by NOS, which exists as three distinct isoforms of NOSs, including endothelial nitric oxide synthase (eNOS), neuronal nitric oxide synthase (nNOS), and iNOS [43]. iNOS is induced by various inflammatory stimuli such as LPS and inflammatory cytokines in macrophages, hepatocytes, and endothelial cells [44-46]. A large amount of NO catalyzed by iNOS plays a key role in the various forms of inflammation and carcinogenesis [46-48].

Noshita et al. [49] investigated the inhibitory activities of 6-MSITC and other synthesized ITCs against LPS-induced NO production using mouse peritoneal macrophages and mouse J774.1 macrophage-like cells. Among the tested ITCs, 6-MSITC indicated the strongest inhibition of $\mathrm{NO}$ production. We also reported that 6-MSITC reduced NO production, and this inhibition depends on the suppression of iNOS expression at the transcriptional level as well as COX-2 expression [12].

3.3. Inflammatory Cytokines/Chemokines. Inflammatory cytokines such as interleukin (IL), interferon (IF), and tumor necrosis factor (TNF) play important roles in the regulation of the immune system [50]. Similar to $\mathrm{PGE}_{2}$ and NO, overproduction of inflammatory cytokines from macrophages causes oxidative stress, systemic inflammation, and cell dysfunction. In addition, chemokines, which are chemotactic cytokines, are well known as multifunctional mediators of gene transcription, cell proliferation, and leukocyte recruitment to inflamed tissues [50]. Chen et al. [51] performed gene expression profiling by DNA microarray in macrophages. Among a total of 22,050 gene probes, LPS upregulated the expression level of 406 genes $(1.8 \%$ of the total gene probes) and downregulated 717 genes $(3.2 \%$ of the total genes probes) by $\geq 3$-fold. The number of genes affected by 6 -MSITC consisted of $58 \%$ of downregulated genes by LPS and $47 \%$ of upregulated genes by LPS. Gene ontology analysis revealed that the gene groups highly affected by 6-MSITC were associated with "inflammatory responses, signal transduction, cytokine activities, hydrolase activity, kinase activity, receptor activity, transferase activity, nucleic acid binding and apoptosis." According to gene profiling and real-time PCR for further confirmation, the upregulation of inflammatory cytokine genes, such as IL- $1 \beta$, IL-6, and TNF, by LPS was reduced by 6-MSITC. 6-MSITC attenuated the expression of IF-inducible genes (IFI1 and IFI47), which are involved in IF-mediated cell proliferation and differentiation. The inductions of IL receptors (IL10ra, IL23ra, and IL4ra) by LPS were also reduced by 6MSITC. These results suggested 6-MSITC inhibition of various inflammatory genes may explain its strong antiinflammatory effects. On the other hand, 6-MSITC restored the expression levels of LPS-reduced CC chemokines
(CCL11 and CCL25), IL-3, and receptors (IL1ra12, IL8ra, TNFRSF23, and TNFRSF4) to control levels. Overall, these data suggest that 6-MSITC might regulate the expression of inflammatory and anti-inflammatory cytokines.

\section{Effect of 6-MSITC on Transcriptional Regulation Involved in Inflammatory Factors}

4.1. Mitogen-Activated Protein Kinase (MAPK). MAPK signaling pathways play a critical role in the regulation of inflammatory response and coordinate the induction of many genes encoding inflammatory factors [52-54]. MAPK has three major subfamily members including extracellularregulated protein kinase (ERK), p38 kinase, and c-Jun $\mathrm{NH}_{2}$ protein kinase (JNK). The activated form of each MAPK phosphorylates and activates other kinases or transcriptional factors, thereby altering the expression of the target genes such as COX-2, iNOS, and inflammatory cytokines [3, 34, 54]. Our data demonstrated that 6-MSITC blocked LPSinduced phosphorylation of all MAPKs and MAPK kinases (MAPKKs) [11]. Furthermore, MAPK-specific inhibitors (U0126 for MEK1/2, SB203580 for p38 kinase, and SP600125 for JNK) demonstrated that LPS-induced COX-2 expression was partially suppressed by the treatment with single inhibitor. However, the combination treatment of two inhibitors markedly reduced COX-2 expression. In particular, cotreatment with three inhibitors completely inhibited COX-2 expression. Thus, these data indicated that three MAPK pathways cooperatively activated COX-2 expression, and 6-MSITC attenuated COX-2 expression by blocking all of three MAPK pathways. On the other hand, only JNKspecific inhibitor SP600125 suppressed LPS-induced iNOS expression, while ERK-specific inhibitor U0126 and p38specific inhibitor SB203580 did not, suggesting that only JNK pathway required iNOS expression, and 6-MSITC might suppress iNOS expression by blocking JNK phosphorylation.

4.2. Activator Protein-1 (AP-1). AP-1, a heterodimer of Jun (c-Jun, Jun B, and JunD) and Fos (cFos, Fos B, Fra-1, and Fra-2), plays an important role in inflammatory responses $[54,55]$. AP-1 is minimally activated under normal physiologic conditions, but is dramatically activated by inflammatory stimuli, like LPS [55]. The activated AP-1 binds to the promoter elements, which regulate the transcription of inflammatory genes such as COX-2, iNOS, TNF- $\alpha$, IL- $1 \beta$, and IL-6 [55]. 6-MSITC completely inhibited LPS-induced phosphorylation of c-Jun, which is a major component of AP-1 in c-Jun/c-Fos heterodimer form [11, 12]. MAPK inhibitors revealed that ERK and JNK signaling pathways cooperatively regulate COX-2 expression by activating AP1 because SP600125 and U0126, but not SB203580, inhibited c-Jun phosphorylation [11]. Moreover, SP600125 suppressed c-Jun phosphorylation and iNOS expression, suggesting that 6-MSITC might inhibit iNOS expression by blocking JNKmediated AP-1 activation (Figure 2) [12].

4.3. CREB and C/EBP. The promoter region of COX-2 gene contains binding sites for CREB and C/EBP. LPS-induced 


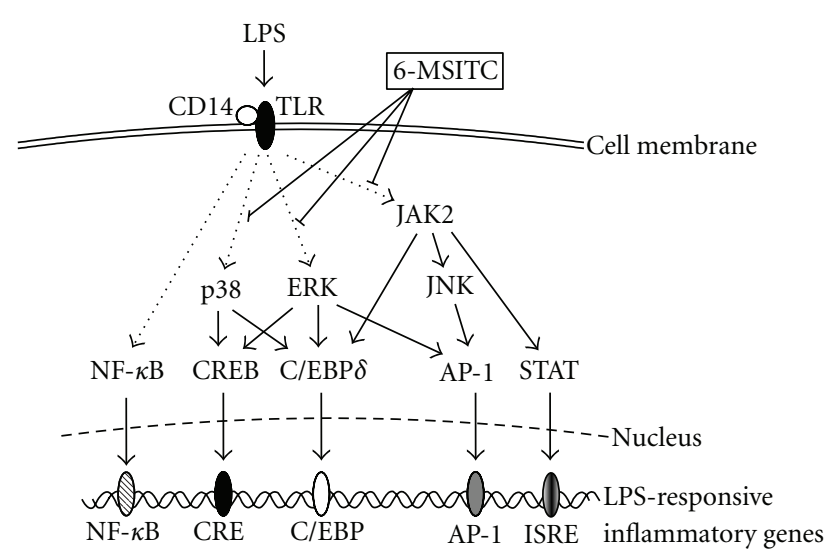

FIGURE 2: Schematic molecular model of 6-MSITC on the suppression of LPS-induced inflammatory factors.

phosphorylation of CREB and nuclear translocation of $\mathrm{C} / \mathrm{EBP}$ can regulate COX-2 gene expression through CRE site and C/EBP site, respectively [56-58]. Several lines of studies have shown that the binding of CREB to CRE site depends on the phosphorylation of CREB $[33,59,60]$, and the binding of C/EBP to COX-2 promoter is preceded by nuclear translocation of C/EBP [28, 56, 61]. 6-MSITC inhibited LPSinduced phosphorylation of CREB [11]. Moreover, LPSinduced expression and nuclear translocation of $\mathrm{C} / \mathrm{EBP} \delta$, but not $\mathrm{C} / \mathrm{EBP} \beta$, were blocked by 6-MSITC [11]. The analysis by MAPK inhibitors demonstrated that ERK and p38 kinase pathways cooperatively regulate COX-2 expression by activating CREB and C/EBP $\delta$ because ERK-specific inhibitor U0126 and p38 kinase-specific inhibitor SB203580 suppressed CREB phosphorylation and C/EBP $\delta$ expression, but JNK-specific inhibitor SP600125 did not. Therefore, 6MSITC blocked LPS-induced COX-2 expression by suppressing ERK and p38 kinase signaling cascades leading to the activation of CREB and C/EBP $\delta$ (Figure 2). The promoter region of iNOS gene also contains binding site for C/EBP. Although 6-MSITC inhibited ERK and p38 kinase signaling cascades leading to $\mathrm{C} / \mathrm{EBP} \delta$, U0126 and SB203580 had no influence on LPS-induced iNOS expression [12]. Hecker et al. reported that $\mathrm{C} / \mathrm{EBP} \beta$ may involve iNOS gene expression synergistically with NF- $\kappa B$ in primary rat hepatocytes [62]. Our data showed that LPS had no influence on the nuclear translocation of $C / \operatorname{EBP} \beta$ [11]. These data suggest that LPS-induced iNOS expression, which was reduced by 6-MSITC, was not involved in $\mathrm{C} / \mathrm{EBP}$ binding site of iNOS gene promoter.

4.4. NF- $\kappa B$. NF- $\kappa B$ is involved in the induction of inflammatory genes and activated by the inflammatory responses during viral and bacterial infections $[63,64]$. Previous analysis has demonstrated that a number of natural occurring compounds suppressed LPS-induced expression of COX-2, iNOS, and inflammatory cytokines by blocking degradation of inhibitor $\kappa \mathrm{B}(\mathrm{I} \kappa \mathrm{B})-\alpha$ in mouse macrophage cells $[56,65$, 66]. However, 6-MSITC had no influence on phosphorylation and degradation of $\mathrm{I} \kappa \mathrm{B}-\alpha$ and nuclear translocation of p65 [11, 12]. Thus, 6-MSITC may inhibit inflammatory factors without the suppression of $\mathrm{I} \kappa \mathrm{B}$ degradation.

4.5. Janus Kinase- (JAK-) Signal Transducers and Activators of Transcription (STAT). The JAK-STAT pathway is an important inflammatory signaling pathway. JAK family, a protein tyrosine kinase (PTK), contains four members, JAK1, JAK2, JAK3, and tyrosine kinase 2 (TYK2), which are differentially regulated in response to various cytokines [67]. Binding of ligands to its receptors activates the phosphorylation of JAK, which subsequently leads to STAT phosphorylation. Phosphorylated STATs translocate to nuclear and regulate the transcription of target genes such as iNOS and COX2 and inflammatory cytokines/chemokines [68-70]. Our data demonstrated that AG490 (JAK2-specific inhibitor) abolished LPS-induced expression of COX-2 (unpublished data) and iNOS [12]. Furthermore, AG490 reduced LPSinduced c-Jun phosphorylation, a major component of AP1 , and $\mathrm{C} / \mathrm{EBP} \delta$ activation. Molecular analysis with AG490 and SP600125 demonstrated that JAK2 acts upstream of JNK leading to AP-1 activation, and JNK cannot regulate the $\mathrm{C} / \mathrm{EBP} \delta$ activation. Moreover, 6-MSITC blocked LPS-induced JAK2 phosphorylation and its downstream pathways. Taken together, JAK2 might upregulate the expression of inflammatory factors through the induction of STAT phosphorylation, C/EBP $\delta$ expression, and JNKmediated AP-1 activation. Moreover, 6-MSITC suppresses LPS-induced JAK2 phosphorylation leading to the induction of inflammatory factors (Figure 2).

\section{Structure-Activity Relationship}

Depending on the length of the alkyl chain of MSITC, there are a number of analogues of MSITC (Figure 3). 4-MSITC, also known as sulforaphane, is a major ITC of broccoli [71], and 2-MSITC and 8-MSITC are artificially synthesized [72]. The inhibitory potency on COX-2 and iNOS expression is increased depending on the alkyl chain elongation $[11,12]$, suggesting that the alkyl chain length is important for the inhibitory activity.

Noshita et al. [49] synthesized a series of ITCs (Figure 3) based on 6-MSITC to check the inhibitory activity against NO production in LPS-induced macrophages. Substitution of ITC group in 6-MSITC with thiocyanate group (1-(methylsulfinyl)-6-thiocyanatohexane, [49]) had no inhibitory effect, indicating the inhibitory action may entirely depend on its ITC group. The alkyl chain elongation in allyl ITCs also increases their lipophilicity ( $\log P$ value), suggesting that the inhibitory potency of inflammatory factors by ITCs may be related to their $\log P$ values. Furthermore, substitution of the methylsulfinyl group in 6MSITC with a formyl (6-isothiocyanatohexanal $[49,73])$, a methylsulfanyl (1-isothiocyanato-6-(methylthio)hexane [49, 74]), or a methyl (n-hexyl ITC [49]), attenuated the inhibitory activity. Polar surface area (PSA) value of these analogues is lower than that of 6-MSITC. In addition, the inhibitory potencies of ITCs showed better correlation with their PSA values rather than their $\log P$ values. Taken 


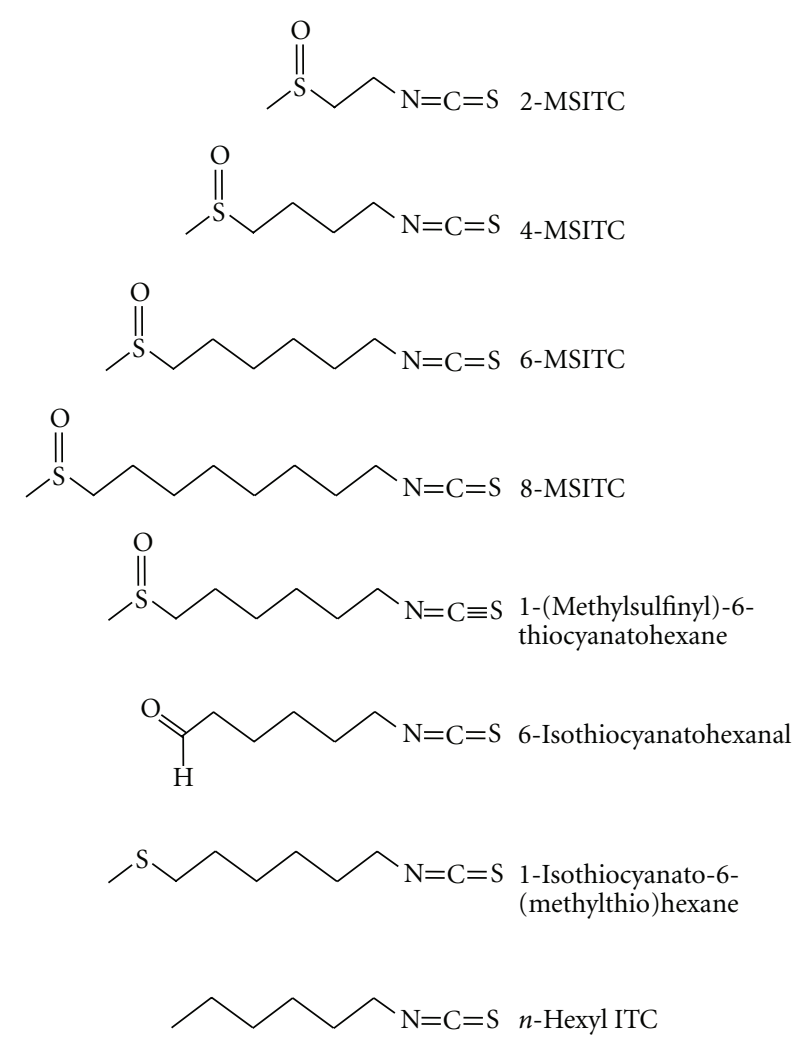

FIGURE 3: Chemical structures of analogues of 6-MSITC. 4-MSITC (sulforaphane), 2-MSITC, and 8-MSITC are analogues of 6-MSITC containing the different length of alkyl chain. 1-(Methylsulfinyl)6-thiocyanatohexane substituted ITC group in 6-MSITC with thiocyanate group. 6-Isothiocyanatohexanal, 1-isothiocyanato-6(methylthio)hexane, and $n$-hexyl ITC substituted the methylsulfinyl group in 6-MSITC with formyl group, methylsulfinyl group, and methyl group, respectively.

together, 6-MSITC has potent biological activity because of its higher PSA value and some degree of $\log P$ value [49].

\section{Cellular Uptake of 6-MSITC}

We investigated the effect of 6-MSITC on the binding of fluorescein-labeled LPS to the LPS receptor by a flow cytometry analysis, and the data suggested that 6-MSITC could not affect the binding of LPS to the receptor in plasma membrane in RAW264 cells (unpublished data). Thus, 6MSITC has no influence on the interaction of LPS receptors. Several studies have revealed the metabolism of ITCs in several cell lines $[4,70,75-77]$. ITCs appear to penetrate cellular membrane by diffusion and rapidly conjugate with intracellular reduced glutathione (GSH) via their ITC group $(-\mathrm{N}=\mathrm{C}=\mathrm{S})$. The methylsulfinyl group $\left(\mathrm{CH}_{3}-\mathrm{S}(=\mathrm{O})-\right)$ and the length of alkyl chain of 6-MSITC might contribute to the cell membrane permeability [49]. GSH is an important intracellular redox buffer that exits as a reduced predominant form, as a disulfide form (GSSG), or as mixed disulfide (GSSR) with protein thiols [78]. The redox status within the cells, reflected by GSH/GSSG [79], has been shown to be relevant for the regulation of inflammatory genes [80]. However, the detailed relationship between GSH-conjugated ITCs and signaling pathways involved in inflammatory factors is not clear. Future studies are needed to elucidate the role of GSH-conjugated 6-MSITC in LPS-induced cellular signaling pathways.

\section{Conclusions}

We have demonstrated that 6-MSITC has inhibited several inflammatory factors such as COX-2, iNOS, and inflammatory cytokines at the transcription factor/promoter levels. MAPK signaling pathways are one of the important pathways involved in inflammatory responses, and 6MSITC suppresses all of three MAPK pathways leading to activation of transcriptional factors. Molecular analysis by MAPK inhibitors revealed the relationship between the transcriptional factors and MAPKs inhibited by 6MSITC. 6-MSITC blocks LPS-induced COX-2 expression by suppressing ERK and p38 kinase signaling cascades leading to the activation of CREB and C/EBP $\delta$, and by inhibiting JNK cascade leading to AP-1 activation. On the other hand, 6-MSITC attenuates iNOS expression mainly by blocking AP-1 activation. In addition, 6-MSITC inhibits JAK2 signaling pathway, which upregulates the expression of inflammatory factors through STAT phosphorylation, $\mathrm{C} / \mathrm{EBP} \delta$ expression, and JNK-mediated AP- 1 activation. We also clarified the structure-activity relationship of MSITC analogues. 6-MSITC has potential usefulness as an antiinflammatory agent because of its higher PSA value and some degree of $\log P$ value.

In recent years, numerous epidemiological and experimental animal studies have shown strong anti-inflammatory and chemopreventive effects of natural products. The elucidation of molecular mechanisms underlying the action of natural compounds may provide further insights into their potential usefulness as anti-inflammatory agents. The further studies on anti-inflammatory properties of 6-MSITC in clinical trial will greatly expand the development of 6MSITC as an anti-inflammatory agent.

\section{Acknowledgments}

This work was funded by "Science and Technology Research Partnership for Sustainable Development (SATREPS)" supported by the Japan Science and Technology Agency (JST), the Japan International Cooperation Agency (JICA), and Japan-China Medical Communication Program from JSPS.

\section{References}

[1] L. Rask, E. Andréasson, B. Ekbom, S. Eriksson, B. Pontoppidan, and J. Meijer, "Myrosinase: gene family evolution and herbivore defense in Brassicaceae," Plant Molecular Biology, vol. 42, no. 1, pp. 93-113, 2000.

[2] R. J. Hopkins, N. M. Van Dam, and J. J. A. Van Loon, "Role of glucosinolates in insect-plant relationships and multitrophic interactions," Annual Review of Entomology, vol. 54, pp. 57-83, 2009. 
[3] K. J. Woo and T. K. Kwon, "Sulforaphane suppresses lipopolysaccharide-induced cyclooxygenase-2 (COX-2) expression through the modulation of multiple targets in COX-2 gene promoter," International Immunopharmacology, vol. 7, no. 13, pp. 1776-1783, 2007.

[4] Y. Zhang, "The molecular basis that unifies the metabolism, cellular uptake and chemopreventive activities of dietary isothiocyanates," Carcinogenesis, vol. 33, no. 1, pp. 2-9, 2012.

[5] S. L. Navarro, F. Li, and J. W. Lampe, "Mechanisms of action of isothiocyanates in cancer chemoprevention: an update," Food \& Function, vol. 2, no. 10, pp. 579-587, 2011.

[6] K. L. Cheung and A. N. Kong, "Molecular targets of dietary phenethyl isothiocyanate and sulforaphane for cancer chemoprevention," AAPS Journal, vol. 12, no. 1, pp. 87-97, 2010.

[7] M. Kojima, "Pungent components and functional ingredient of wasabi," Food Process, vol. 23, pp. 32-35, 1988.

[8] K. Isshiki and K. Tokuoka, "Allyl isothiocyanate and wholesomeness of food," Japanese Journal of Food Microbiology, vol. 12, pp. 1-6, 1993.

[9] H. Kumagai, N. Kashima, T. Seki, H. Sakurai, K. Ishii, and T. Ariga, "Analysis of volatile components in essential oil of upland Wasabi and their inhibitory effects on platelet aggregation," Bioscience, Biotechnology and Biochemistry, vol. 58, no. 12, pp. 2131-2135, 1994.

[10] N. Tanida, A. Kawaura, A. Takahashi, K. Sawada, and T. Shimoyama, "Suppressive effect of Wasabi (pungent Japanese spice) on gastric carcinogenesis induced by MNNG in rats," Nutrition and Cancer, vol. 16, no. 1, pp. 53-58, 1991.

[11] T. Uto, M. Fujii, and D. X. Hou, "Inhibition of lipopolysaccharide-induced cyclooxygenase-2 transcription by 6-(methylsulfinyl) hexyl isothiocyanate, a chemopreventive compound from Wasabia japonica (Miq.) Matsumura, in mouse macrophages," Biochemical Pharmacology, vol. 70, no. 12, pp. 1772-1784, 2005.

[12] T. Uto, M. Fujii, and D. X. Hou, "6-(Methylsulfinyl)hexyl isothiocyanate suppresses inducible nitric oxide synthase expression through the inhibition of Janus kinase 2-mediated JNK pathway in lipopolysaccharide-activated murine macrophages," Biochemical Pharmacology, vol. 70, no. 8, pp. 1211-1221, 2005.

[13] T. Uto, M. Fujii, and D. X. Hou, "Effects of 6-(methylsulfinyl) hexyl isothiocyanate on cyclooxygenase-2 expression induced by lipopolysaccharide, interferon-gamma and 12-Otetradecanoylphorbol-13-acetate," Oncology Reports, vol. 17, no. 1, pp. 233-238, 2007.

[14] N. Hasegawa, Y. Matsumoto, A. Hoshino, and K. Iwashita, "Comparison of effects of Wasabia japonica and allyl isothiocyanate on the growth of four strains of Vibrio parahaemolyticus in lean and fatty tuna meat suspensions," International Journal of Food Microbiology, vol. 49, no. 1-2, pp. 27-34, 1999.

[15] Y. Morimitsu, K. Hayashi, Y. Nakagawa et al., "Antiplatelet and anticancer isothiocyanates in Japanese domestic horseradish, Wasabi," Mechanisms of Ageing and Development, vol. 116, no. 2-3, pp. 125-134, 2000.

[16] D. X. Hou, M. Fukuda, M. Fujii, and Y. Fuke, "Induction of NADPH:quinone oxidoreductase in murine hepatoma cells by methylsulfinyl isothiocyanates: methyl chain length-activity study," International Journal of Molecular Medicine, vol. 6, no. 4, pp. 441-444, 2000.

[17] D. X. Hou, M. Fukuda, M. Fujii, and Y. Fuke, "Transcriptional regulation of nicotinamide adenine dinucleotide phosphate: quinone oxidoreductase in murine hepatoma cells by 6 (methylsufinyl)hexyl isothiocyanate, an active principle of wasabi (Eutrema wasabi Maxim)," Cancer Letters, vol. 161, no. 2, pp. 195-200, 2000.

[18] D. X. Hou, Y. Korenori, S. Tanigawa et al., "Dynamics of Nrf2 and Keap1 in ARE-mediated NQO1 expression by wasabi 6(methylsulfinyl)hexyl isothiocyanate," Journal of Agricultural and Food Chemistry, vol. 59, no. 22, pp. 11975-11882.

[19] K. Ina, H. Ina, M. Ueda, A. Yagi, and I. Kishima, “ $\omega$ Methylthioalkyl isothiocyanates in Wasabi," Agricultural Biology and Chemistry, vol. 53, no. 2, pp. 537-538, 1989.

[20] H. Etoh, A. Nishimura, R. Takasawa et al., “ $\omega$ Methylsulfinylalkyl isothiocyanates in wasabi, Wasabia japonica Matsum," Agricultural Biology and Chemistry, vol. 54, no. 6, pp. 1587-1589, 1990.

[21] M. Hara, K. Mochizuki, S. Kaneko et al., "Changes in pungent components of two Wasabia japonica Matsum. cultivars during the cultivation period," Food Science and Technology Research, vol. 9, no. 3, pp. 288-291, 2003.

[22] H. Ono, K. Adachi, Y. Fuke, and K. Shinohara, "Purification and structural analysis of substances in wasabi (Eutrema wasabi maxim.) that suppress the growth of MKN-28 human stomach cancer cells," Nippon Shokuhin Kagaku Kogaku Kaishi, vol. 43, no. 10, pp. 1092-1097, 1996.

[23] Y. Morimitsu, Y. Nakagawa, K. Hayashi et al., "A sulforaphane analogue that potently activates the Nrf2-dependent detoxification pathway," The Journal of Biological Chemistry, vol. 277, no. 5, pp. 3456-3463, 2002.

[24] M. D. Maines, "The heme oxygenase system: a regulator of second messenger gases," Annual Review of Pharmacology and Toxicology, vol. 37, pp. 517-554, 1997.

[25] L. Boscá, M. Zeini, P. G. Través, and S. Hortelano, "Nitric oxide and cell viability in inflammatory cells: a role for $\mathrm{NO}$ in macrophage function and fate," Toxicology, vol. 208, no. 2, pp. 249-258, 2005.

[26] Z. Zhu, S. Zhong, and Z. Shen, "Targeting the inflammatory pathways to enhance chemotherapy of cancer," Cancer Biology and Therapy, vol. 12, no. 2, pp. 95-105, 2011.

[27] B. Hinz and K. Brune, "Cyclooxygenase-2-0 years later," Journal of Pharmacology and Experimental Therapeutics, vol. 300, no. 2, pp. 367-375, 2002.

[28] R. G. Molloy, J. A. Mannick, and M. L. Rodrick, "Cytokines, sepsis and immunomodulation," British Journal of Surgery, vol. 80, no. 3, pp. 289-297, 1993.

[29] C. D. Funk, L. B. Funk, M. E. Kennedy, A. S. Pong, and G. A. Fitzgerald, "Human platelet/erythroleukemia cell prostaglandin $\mathrm{G} / \mathrm{H}$ synthase: cDNA cloning, expression, and gene chromosomal assignment," FASEB Journal, vol. 5, no. 9, pp. 2304-2312, 1991.

[30] S. L. Hempel, M. M. Monick, and G. W. Hunninghake, "Lipopolysaccharide induces prostaglandin $\mathrm{H}$ synthase-2 protein and mRNA in human alveolar macrophages and blood monocytes," Journal of Clinical Investigation, vol. 93, no. 1, pp. 391-396, 1994.

[31] L. J. Crofford, R. L. Wilder, A. P. Ristimaki et al., "Cyclooxygenase-1 and -2 expression in rheumatoid synovial tissues. Effects of interleukin-1 $\beta$, phorbol ester, and corticosteroids," Journal of Clinical Investigation, vol. 93, no. 3, pp. 1095-1101, 1994.

[32] J. R. Mestre, P. J. Mackrell, D. E. Rivadeneira, P. P. Stapleton, T. Tanabe, and J. M. Daly, "Redundancy in the signaling pathways and promoter elements regulating cyclooxygenase- 2 gene expression in endotoxin-treated macrophage/monocytic cells," The Journal of Biological Chemistry, vol. 276, no. 6, pp. 3977-3982, 2001. 
[33] M. Caivano, B. Gorgoni, P. Cohen, and V. Poli, "The induction of cyclooxygenase- 2 mRNA in macrophages is biphasic and requires both CCAAT enhancer-binding protein beta $(\mathrm{C} / \mathrm{EBP}$ beta) and C/EBP delta transcription factors," The Journal of Biological Chemistry, vol. 276, no. 52, pp. 48693-48701, 2001.

[34] H. Inoue, C. Yokoyama, S. Hara, Y. Tone, and T. Tanabe, "Transcriptional regulation of human prostaglandinendoperoxide synthase-2 gene by lipopolysaccharide and phorbol ester in vascular endothelial cells. Involvement of both nuclear factor for interleukin-6 expression site and cAMP response element," The Journal of Biological Chemistry, vol. 270, no. 42, pp. 24965-24971, 1995.

[35] H. Inoue, T. Nanayama, S. Hara, C. Yokoyama, and T. Tanabe, "The cyclic AMP response element plays an essential role in the expression of the human prostaglandin-endoperoxide synthase 2 gene in differentiated U937 monocytic cells," FEBS Letters, vol. 350, no. 1, pp. 51-54, 1994.

[36] H. Inoue and T. Tanabe, "Transcriptional role of the nuclear factor $\kappa \mathrm{B}$ site in the induction by lipopolysaccharide and suppression by dexamethasone of cyclooxygenase-2 in U937 cells," Biochemical and Biophysical Research Communications, vol. 244, no. 1, pp. 143-148, 1998.

[37] S. Moncada, R. M. J. Palmer, and E. A. Higgs, "Nitric oxide: physiology, pathophysiology, and pharmacology," Pharmacological Reviews, vol. 43, no. 2, pp. 109-142, 1991.

[38] W. K. Alderton, C. E. Cooper, and R. G. Knowles, "Nitric oxide synthases: structure, function and inhibition," Biochemical Journal, vol. 357, no. 3, pp. 593-615, 2001.

[39] K. D. Kröncke, K. Fehsel, and V. Kolb-Bachofen, "Inducible nitric oxide synthase in human diseases," Clinical and Experimental Immunology, vol. 113, no. 2, pp. 147-156, 1998.

[40] R. B. Lorsbach, W. J. Murphy, C. J. Lowenstein, S. H. Snyder, and S. W. Russell, "Expression of the nitric oxide synthase gene in mouse macrophages activated for tumor cell killing. Molecular basis for the synergy between interferon- $\gamma$ and lipopolysaccharide," The Journal of Biological Chemistry, vol. 268, no. 3, pp. 1908-1913, 1993.

[41] R. Korhonen, A. Lahti, H. Kankaanranta, and E. Moilanen, "Nitric oxide production and signaling in inflammation," Current Drug Targets, vol. 4, no. 4, pp. 471-479, 2005.

[42] H. Ohshima and H. Bartsch, "Chronic infections and inflammatory processes as cancer risk factors: possible role of nitric oxide in carcinogenesis," Mutation Research, vol. 305, no. 2, pp. 253-264, 1994.

[43] R. M. J. Palmer, D. S. Ashton, and S. Moncada, "Vascular endothelial cells synthesize nitric oxide from L-arginine," Nature, vol. 333, no. 6174, pp. 664-666, 1988.

[44] C. Nathan and Q. W. Xie, "Nitric oxide synthases: roles, tolls, and controls," Cell, vol. 78, no. 6, pp. 915-918, 1994.

[45] Y. Kobayashi, "The regulatory role of nitric oxide in proinflammatory cytokine expression during the induction and resolution of inflammation," Journal of Leukocyte Biology, vol. 88, no. 6, pp. 1157-1162, 2010.

[46] J. MacMicking, Q. W. Xie, and C. Nathan, "Nitric oxide and macrophage function," Annual Review of Immunology, vol. 15, pp. 323-350, 1997.

[47] H. Maeda and T. Akaike, "Nitric oxide and oxygen radicals in infection, inflammation and cancer," Biochemistry (Moscow), vol. 63, no. 7, pp. 854-865, 1998.

[48] P. K. Lala and C. Chakraborty, "Role of nitric oxide in carcinogenesis and tumour progression," The Lancet Oncology, vol. 2, no. 3, pp. 149-156, 2001.
[49] T. Noshita, Y. Kidachi, H. Funayama, H. Kiyota, H. Yamaguchi, and K. Ryoyama, "Anti-nitric oxide production activity of isothiocyanates correlates with their polar surface area rather than their lipophilicity," European Journal of Medicinal Chemistry, vol. 44, no. 12, pp. 4931-4936, 2009.

[50] A. D. Luster, "Mechanisms of disease: chemokineschemotactic cytokines that mediate inflammation," The New England Journal of Medicine, vol. 338, no. 7, pp. 436-445, 1998.

[51] J. Chen, T. Uto, S. Tanigawa, T. Yamada-Kato, M. Fujii, and D. X. Hou, "Microarray-based determination of antiinflammatory genes targeted by 6-(methylsulfinyl)hexyl isothiocyanate in macrophages," Experimental and Therapeutic Medicine, vol. 1, no. 1, pp. 33-40, 2010.

[52] C. Tsatsanis, A. Androulidaki, M. Venihaki, and A. N. Margioris, "Signalling networks regulating cyclooxygenase-2," International Journal of Biochemistry and Cell Biology, vol. 38, no. 10, pp. 1654-1661, 2006.

[53] P. Huang, J. Han, and L. Hui, "MAPK signaling in inflammation-associated cancer development," Protein and Cell, vol. 1, no. 3, pp. 218-226, 2010.

[54] S. K. Dower and E. E. Qwarnstrom, "Signalling networks, inflammation and innate immunity," Biochemical Society Transactions, vol. 31, no. 6, pp. 1462-1471, 2003.

[55] T. C. Hsu, M. R. Young, J. Cmarik, and N. H. Colburn, "Activator protein 1 (AP-1)- and nuclear factor $\kappa \mathrm{B}$ (NF$\kappa \mathrm{B})$-dependent transcriptional events in carcinogenesis," Free Radical Biology and Medicine, vol. 28, no. 9, pp. 1338-1348, 2000.

[56] A. K. Lee, S. H. Sung, Y. C. Kim, and S. G. Kim, "Inhibition of lipopolysaccharide-inducible nitric oxide synthase, TNF$\alpha$ and COX-2 expression by sauchinone effects on I- $\kappa \mathrm{B} \alpha$ phosphorylation, C/EBP and AP-1 activation," British Journal of Pharmacology, vol. 139, no. 1, pp. 11-20, 2003.

[57] B. Thomas, F. Berenbaum, L. Humbert et al., "Critical role of $\mathrm{C} / \mathrm{EBP} \delta$ and $\mathrm{C} / \mathrm{EBP} \beta$ factors in the stimulation of the cyclooxygenase- 2 gene transcription by interleukin- $1 \beta$ in articular chondrocytes," European Journal of Biochemistry, vol. 267, no. 23, pp. 6798-6809, 2000.

[58] F. D’Acquisto, T. Iuvone, L. Rombolà, L. Sautebin, M. Di Rosa, and R. Carnuccio, "Involvement of NF- $\kappa$ B in the regulation of cyclooxygenase-2 protein expression in LPS-stimulated J774 macrophages," FEBS Letters, vol. 418, no. 1-2, pp. 175-178, 1997.

[59] K. Subbaramaiah, P. A. Cole, and A. J. Dannenberg, "Retinoids and carnosol suppress cyclooxygenase- 2 transcription by CREB-binding protein/p300-dependent and -independent mechanisms," Cancer Research, vol. 62, no. 9, pp. 2522-2530, 2002.

[60] A. G. Eliopoulos, C. D. Dumitru, C. C. Wang, J. Cho, and P. $\mathrm{N}$. Tsichlis, "Induction of COX-2 by LPS in macrophages is regulated by Tpl2-dependent CREB activation signals," EMBO Journal, vol. 21, no. 18, pp. 4831-4840, 2002.

[61] Y. H. Cho, C. H. Lee, and S. G. Kim, "Potentiation of lipopolysaccharide-inducible cyclooxygenase 2 expression by C2-ceramide via c-Jun $\mathrm{N}$-terminal kinase-mediated activation of CCAAT/enhancer binding protein $\beta$ in macrophages," Molecular Pharmacology, vol. 63, no. 3, pp. 512-523, 2003.

[62] M. Hecker, C. Preiß, and V. B. Schini-Kerth, "Induction by staurosporine of nitric oxide synthase expression in vascular smooth muscle cells: role of NF- $\kappa \mathrm{B}, \mathrm{CREB}$ and $\mathrm{C} / \mathrm{EBP} \beta$," British Journal of Pharmacology, vol. 120, no. 6, pp. 1067-1074, 1997. 
[63] J. Marks-Konczalik, S. C. Chu, and J. Moss, "Cytokinemediated transcriptional induction of the human inducible nitric oxide synthase gene requires both activator protein 1 and nuclear factor $\kappa \mathrm{B}$-binding sites," The Journal of Biological Chemistry, vol. 273, no. 35, pp. 22201-22208, 1998.

[64] Q. W. Xie, Y. Kashiwabara, and C. Nathan, "Role of transcription factor NF- $\kappa \mathrm{B} / \mathrm{Rel}$ in induction of nitric oxide synthase," The Journal of Biological Chemistry, vol. 269, no. 7, pp. 47054708, 1994.

[65] C. S. Kim, T. Kawada, B. S. Kim et al., "Capsaicin exhibits antiinflammatory property by inhibiting $\mathrm{I} \kappa \mathrm{B}-\alpha$ degradation in LPS-stimulated peritoneal macrophages," Cellular Signalling, vol. 15, no. 3, pp. 299-306, 2003.

[66] Y. C. Liang, Y. T. Huang, S. H. Tsai, S. Y. Lin-Shiau, C. F. Chen, and J. K. Lin, "Suppression of inducible cyclooxygenase and inducible nitric oxide synthase by apigenin and related flavonoids in mouse macrophages," Carcinogenesis, vol. 20, no. 10, pp. 1945-1952, 1999.

[67] K. Imada and W. J. Leonard, "The Jak-STAT pathway," Molecular Immunology, vol. 37, no. 1-2, pp. 1-11, 2000.

[68] P. Kovarik, M. Mangold, K. Ramsauer et al., "Specificity of signaling by STAT1 depends on SH2 and C-terminal domains that regulate Ser727 phosphorylation, differentially affecting specific target gene expression," EMBO Journal, vol. 20, no. 12, pp. 91-100, 2001.

[69] R. W. Ganster, B. S. Taylor, L. Shao, and D. A. Geller, "Complex regulation of human inducible nitric oxide synthase gene transcription by Stat 1 and NF- $\kappa \mathrm{B}$," Proceedings of the National Academy of Sciences of the United States of America, vol. 98, no. 15, pp. 8638-8643, 2001.

[70] Z. Wen, Z. Zhong, and J. E. Darnell Jr., "Maximal activation of transcription by Stat 1 and Stat 3 requires both tyrosine and serine phosphorylation," Cell, vol. 82, no. 2, pp. 241-250, 1995.

[71] Y. Zhang, P. Talalay, C. G. Cho, and G. H. Posner, "A major inducer of anticarcinogenic protective enzymes from broccoli: isolation and elucidation of structure," Proceedings of the National Academy of Sciences of the United States of America, vol. 89, no. 6, pp. 2399-2403, 1992.

[72] T. Nomura, S. Shinoda, T. Yamori et al., "Selective sensitivity to wasabi-derived 6-(methylsulfinyl)hexyl isothiocyanate of human breast cancer and melanoma cell lines studied in vitro," Cancer Detection and Prevention, vol. 29, no. 2, pp. 155-160, 2005.

[73] S. Löhr, C. Jacobi, A. Johann, G. Gottschalk, and A. De Meijere, "Cyclopropyl building blocks in organic synthesis, 57convenient syntheses and biological activity of novel $\omega$-trans(bicyclopropyl)-and $\omega$-(bicyclopropylidenyl)-substituted fatty acids and their derivatives," European Journal of Organic Chemistry, no. 17, pp. 2979-2989, 2000.

[74] T. J. Ding, L. Zhou, and X. P. Cao, "A facile and green synthesis of sulforaphane," Chinese Chemical Letters, vol. 17, no. 9, pp. 1152-1154, 2006.

[75] Y. Zhang and P. Talalay, "Mechanism of differential potencies of isothiocyanates as inducers of anticarcinogenic Phase 2 enzymes," Cancer Research, vol. 58, no. 20, pp. 4632-4639, 1998.

[76] Y. Zhang, "Role of glutathione in the accumulation of anticarcinogenic isothiocyanates and their glutathione conjugates by murine hepatoma cells," Carcinogenesis, vol. 21, no. 6, pp. 1175-1182, 2000.

[77] L. Mi, A. J. Di Pasqua, and F. L. Chung, "Proteins as binding targets of isothiocyanates in cancer prevention," Carcinogenesis, vol. 32, no. 10, pp. 1405-1413, 2011.
[78] S. M. Deneke and B. L. Fanburg, "Regulation of cellular glutathione," American Journal of Physiology, vol. 257, no. 4, pp. L163-L173, 1989.

[79] I. A. Cotgreave and R. G. Gerdes, "Recent trends in glutathione biochemistry-glutathione-protein interactions: a molecular link between oxidative stress and cell proliferation?" Biochemical and Biophysical Research Communications, vol. 242, no. 1, pp. 1-9, 1998.

[80] I. Rahman and W. MacNee, "Regulation of redox glutathione levels and gene transcription in lung inflammation: therapeutic approaches," Free Radical Biology and Medicine, vol. 28, no. 9, pp. 1405-1420, 2000. 

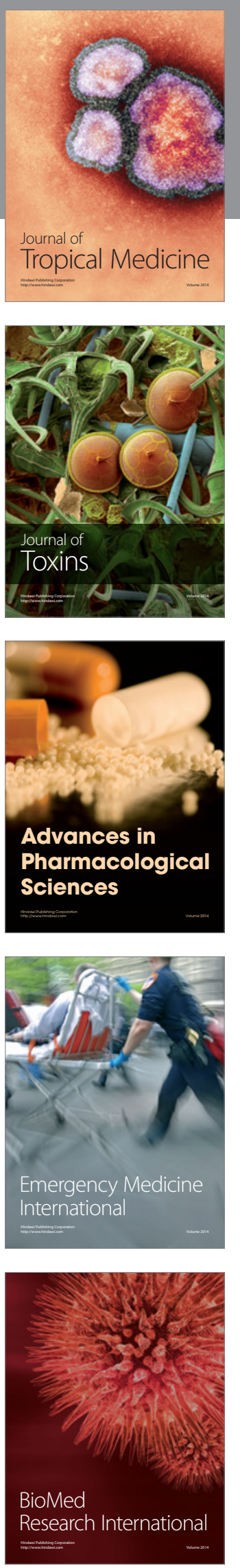
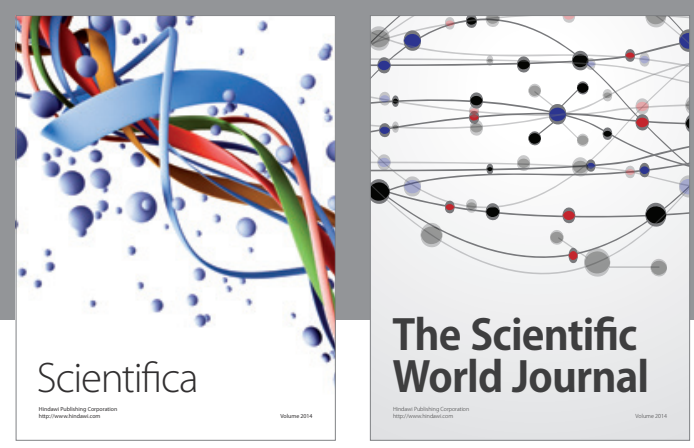

The Scientific World Journal
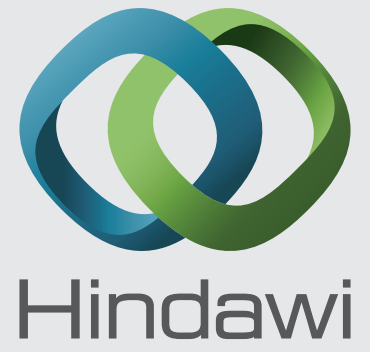

Submit your manuscripts at

http://www.hindawi.com
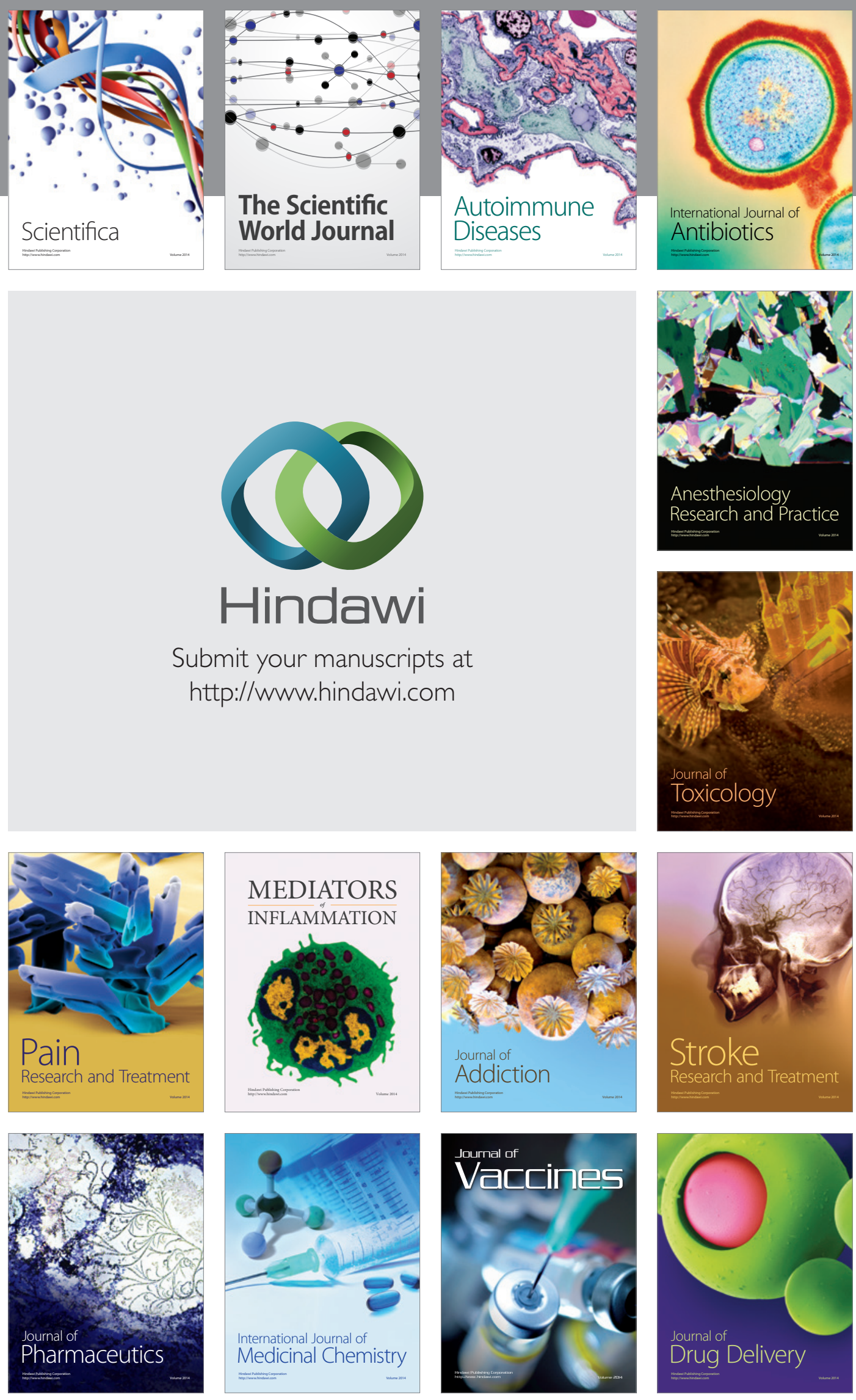